\title{
Reflets
}

Revue ontaroise d'intervention sociale et communautaire

\section{Résumés des mémoires, Universités d'Ottawa et Laurentienne}

Madeleine Aumond, J. Derrick Barnes, Francine Beaulieu, Claude Bouchard, Chantal Bradley, Nathalie Cantin-Plante, Catherine Landriault, Sylvie Lemaire, Aline A. Lévesque, Michèle Mayer, Jocelyne McKellar, Nathalie L. Ouellette et Sylvie Rivard

Volume 5, numéro 1, printemps 1999

Pratiques et développement économique communautaire

URI : https://id.erudit.org/iderudit/026265ar

DOI : https://doi.org/10.7202/026265ar

Aller au sommaire du numéro

Éditeur(s)

Reflets : Revue ontaroise d'intervention sociale et communautaire

ISSN

1203-4576 (imprimé)

1712-8498 (numérique)

Découvrir la revue

Citer ce document

Aumond, M., Barnes, J. D., Beaulieu, F., Bouchard, C., Bradley, C., Cantin-Plante, N., Landriault, C., Lemaire, S., Lévesque, A. A., Mayer, M., McKellar, J., Ouellette, N. L. \& Rivard, S. (1999). Résumés des mémoires, Universités d’Ottawa et Laurentienne. Reflets, 5(1), 226-237. https://doi.org/10.7202/026265ar

Tous droits réservés (C) Reflets : Revue ontaroise d'intervention sociale et communautaire, 1999
Ce document est protégé par la loi sur le droit d'auteur. L'utilisation des services d'Érudit (y compris la reproduction) est assujettie à sa politique d'utilisation que vous pouvez consulter en ligne. 


\title{
La prévention en amont des signalements. Les Centres Jeunesse Outaouais, bureau de Maniwaki
}

\author{
Madeleine Aumond \\ École de service social, Université Laurentienne
}

L'état actuel des connaissances en matière de prévention à la protection de la jeunesse, les préoccupations exprimées par les représentants de l'organisme et le fait que l'intervenant soit le principal instigateur dans la prévention, nous amènent à nous intéresser de manière plus spécifique au processus de l'intervention en amont des signalements. L'objectif général de cette étude est de décrire l'intervention en prévention en amont des signalements. Plus spécifiquement, les objectifs poursuivis par cette étude sont les suivants : décrire le processus (table d'orientation) de l'action proactive en amont des signalements; définir la fonction des tables d'orientations; décrire les caractéristiques socio-démographiques des enfants et des familles; décrire les problématiques; décrire les rôles des principaux acteurs (intervenants DPJ) dans l'intervention et finalement, décrire les principales étapes de l'orientation impliquant le jeune et sa famille.

\section{La rénovation urbaine de la basse-ville d'Ottawa: la théorie, les politiques et les effets sur la population francophone}

\author{
J. Derrick Barnes \\ École de service social, Université d'Ottawa
}

En dépit des impacts importants de la rénovation urbaine de la Basse-Ville d'Ottawa des années 1960 pour la communauté francophone qui y habitait, il existe très peu de recherche formelle 
sur ce sujet. Ce mémoire vise donc à mettre en contexte ce projet de rénovation urbaine. Par le biais d'une analyse documentaire, deux grands axes ont été examinés.

Le premier axe explore les courants de pensée véhiculés à l'époque de ce projet de rénovation urbaine et les attitudes face à la vie urbaine. Le deuxième axe situe la rénovation urbaine dans le contexte des interventions gouvernementales dans le domaine $\mathrm{du}$ logement. Certains thèmes se dégagent de l'analyse de ces deux axes: il y avait une méfiance face à la vie urbaine, la vie rurale étant perçue comme étant plus saine moralement et physiquement; les interventions gouvernementales étaient le résultat d'une logique keynésienne et n'ont pas nécessairement privilégié les voeux populaires. Suite à l'identification de ces thèmes, ce texte examine quelques effets de la rénovation urbaine sur la population francophone de la Basse-Ville.

L'analyse effectuée démontre que les francophones n'avaient pas une grande voix dans l'évolution du projet de rénovation. Désavantagés par leur situation défavorable d'éducation, de revenu et par l'isolement engendré par leur langue, les francophones ont perdu leurs droits à un "chez eux» leur appartenant. Les changements dans leur quartier ont été bouleversants sur le plan physique et ont engendré des impacts importants sur les relations d'entraide et sur le sens d'appartenance.

\section{L'accessibilité aux services de soutien à domicile pour les personnes âgées francophones de la municipalité de Sudbury}

\section{Francine Beaulieu}

École de service social, Université Laurentienne

Une étude de l'accessibilité aux services de soutien à domicile pour les personnes âgées francophones a été réalisée auprès de personnes âgées de la Municipalité de Sudbury. Cette recherche vise à identifier 
les difficultés rencontrées par les personnes âgées lorsqu'elles ont eu recours aux services de soutien à domicile offerts dans le cadre du Programme de services de santé et de soutien personnel et du Programme des services de soutien communautaire

L'identification des obstacles à l'accessibilité de soutien à domicile a été réalisée en effectuant une analyse qualitative de contenu. L'analyse de l'accessibilité aux services s'est appuyée sur la théorie de la valorisation des rôles sociaux.Au cours de la recherche,l'auteure a réalisé des entrevues semi-structurées auprès de vingt-trois (23) personnes âgées francophones, au sein de la Municipalité régionale de Sudbury, qui recevaient des services de soutien à domicile.

Les principales difficultés vécues par les personnes âgées sont les suivantes: non reconnaissance de l'apport du réseau naturel par les réseaux formels de service; instabilité dans le financement des organismes qui relèvent du Programme de soutien communautaire et gamme incomplète de services dans ce programme; disponibilité inégale des services en français; méconnaissance des droits pour les personnes âgées de recevoir des services en français; insuffisance de coordination et de continuité entre les programmes et le réseau naturel des personnes âgées; horaire trop restreint des services offerts dans le Programme de santé et de soutien personnel; absence de ressources masculines au service d'aide familiale.

\section{L'analyse des besoins de formation des francophones oeuvrant dans les Sociétés d'aide à l'enfance de l'Ontario}

\section{Claude Bouchard}

École de service social, Université Laurentienne

Dans cet essai, l'auteur étudie les résultats d'une analyse des besoins de formation des francophones oeuvrant dans les Sociétés de l'aide à l'enfance (SAE) de l'Ontario. Cette étude fut menée dans le 
cadre d'un stage pratique à l'Association ontarienne des sociétés de l'aide à l'enfance, entre novembre 1996 et février 1997.

S'appuyant sur la méthode du groupe focus, l'essai révèle que les réponses aux questions de départ de l'auteur confirment la pratique discursive politiquement correcte de l'organisme de stage et la centration culturelle véhiculée par le système actuel de formation en matière de bien-être de l'enfance dont elle est responsable.

L'auteur documente sa compréhension de la structure organisationnelle du milieu de stage et des éléments dynamiques de ce qui fut son champ de pratique en lien avec les résultats obtenus lors des groupes focus.

Cet essai propose enfin une évaluation du plan d'action soumis à l'Association ontarienne des SAE découlant des besoins identifiés par les francophones du réseau. L'auteur tente d'en interpréter les impacts et d'en saisir les indicateurs futurs du changement.

\title{
«Je ne suis pas une étiquette, je suis une personne»: la problématique de l'étiquetage social et la réforme de santé mentale en Ontario
}

\author{
Chantal Bradley \\ École de service social, Université d'Ottawa
}

Ce mémoire examine certains enjeux rattachés a l'étiquetage social en santé mentale. Après avoir cerné la réalité de l'étiquetage en santé mentale, ses conséquences sociales, en particulier le rejet et l'exclusion sociale.

Nous avons choisi d'étudier cette problématique plus spécifiquement dans le cadre des perspectives biomédicale et antipsychiatrique en santé mentale. Nous concluons avec l'examen du concept de l'exclusion sociale en nous référant a son opposé, l'inclusion sociale. La méthode de recherche utilisée est de type documentaire et qualitatif. 
En dernier lieu, nous examinons plus précisément la réforme de santé mentale en Ontario en tenant compte de la problématique de l'étiquetage social proposée dans ce travail.

En guise de conclusion, nous suggérons quelques pistes de réflexion sur les interventions sociales possibles pour contrer les effets pervers de l'étiquetage.

\title{
La conciliation du travail et de la famille: une problématique particulière pour les femmes monoparentales
}

\author{
Nathalie Cantin-Plante \\ École de service social, Université d'Ottawa
}

Les femmes monoparentales vivent dans une grande proportion dans la pauvreté. Lorsqu'elles sont responsables d'enfants de moins de 6 ans, les difficultés liées à la conciliation du travail et de la famille se font particulièrement criantes. Ainsi, elles courent davantage de risques de se trouver piégées dans un cercle vicieux où, alors que le marché du travail ne leur permet pas de répondre aux besoins de leur famille à cause d'un manque de ressources financières et de la difficulté à concilier travail et famille, elles se trouvent à dépendre d'un État qui, lui, les pousse à retourner le plus rapidement possible sur ce même marché du travail qui les avait d'abord exclues. En effet, des conditions précaires d'emploi et un manque de ressources telles que des services de garde abordables et accessibles rendent particulièrement problématique pour les femmes monoparentales la conciliation de la vie professionnelle et de la vie familiale. Alors que l'État québécois tente d'offrir des mesures de réinsertion sur le marché de l'emploi et de rendre plus accessibles les services de garde, le marché du travail, lui, ne semble pas en voie d'accorder une plus grande reconnaissance aux responsabilités familiales des travailleuses et 
travailleurs. L'économie sociale se présente alors comme une façon de travailler autrement et de créer des services mieux adaptés aux besoins des différentes communautés.

\section{Le DSM : une entreprise colossale mais fragile. Recension des écrits critiques sur le manuel diagnostique de psychiatrie}

\section{Catherine Landriault}

École de service social, Université d'Ottawa

En 1994, l'Association américaine de psychiatrie a publié la $4^{e}$ édition du Manuel diagnostique et statistique des troubles mentaux (DSM-IV). Ce manuel présente une classification des troubles mentaux qui est aujourd'hui reconnue internationalement et qui exerce une influence importante sur la recherche et la pratique dans le champ de la santé mentale.

Le DSM comprend des enjeux complexes qui impliquent les professionnels de la santé mentale, le système de santé et les personnes qui souffrent de troubles mentaux.Après avoir examiné les enjeux du diagnostic psychiatrique et étudié le développement du DSM dans son contexte historique et social, il s'agit de porter un regard critique sur le manuel, en analysant de nombreux écrits critiques portant sur le sujet. Le processus de construction du manuel est tout d'abord examiné et démontre le rôle qu'y jouent les forces politiques et sociales. Ensuite, divers principes et concepts sous-jacents au système de classification son analysés, soit le choix d'une approche descriptive et catégorielle, la fiabilité des diagnostics psychiatriques et la validité du concept de trouble mental. La prise en considération des facteurs sociaux et culturels dans le processus diagnostique est enfin décrite et critiquée. La remise en question du DSM révèle ainsi que le trouble mental est en fait un construit professionnel et social. La conclusion examine 
les implications du DSM pour la pratique en ouvrant sur une autre façon d'intervenir, qui peut s'avérer particulièrement pertinente pour le service social: la narrativité.

\title{
La monoparentalité, c'est une question de survie, de solitude et d'autonomie
}

\author{
Sylvie Lemaire \\ Ecole de service social, Université d'Ottawa
}

Même si les familles monoparentales sont de plus en plus nombreuses, la plupart des recherches se penchent sur les répercussions négatives observées chez les enfants à la suite d'un divorce ou d'une séparation. Ces études et celles qui comparent les familles monoparentales et bi-parentales sont venues soutenir la norme familiale dominante, tout en contribuant au stigma rattaché à la famille monoparentale. Notre étude a examiné tant les aspects positifs que négatifs des familles monoparentales dirigées par une femme. Un deuxième objectif initial était d'identifier les stratégies utilisées par celles-ci pour surmonter les obstacles rencontrés. Nous avons eu recours à l'analyse par théorisation ancrée comme méthode de cueillette des données, ce qui amène habituellement une modification des questions d'origines. Notre étude s'est alors penchée sur les transitions que vivent ces femmes entre la fin de vie de couple et la situation où elles sont seules avec leurs enfants. Nous avons réalisé notre étude exploratoire par l'entremise d'entrevues semi-dirigées auprès de trois femmes et de deux observations participantes auprès de groupes de femmes monoparentales.

Les résultats indiquent que les femmes qui vivent un divorce ou une séparation passent à travers trois phases de transition que nous avons nommées: «J'ai peur d'être seule, je suis seule et j'aime être seule». La première phase parait être plus longue et plus compliquée que les autres à cause des obstacles d'ordres 
psychologiques, économiques et socio-culturels qui peuvent retarder la prise de décision initiale de mettre fin à la vie de couple. Les stratégies utilisées pour surmonter les obstacles different selon la phase de transition où se situe la femme. Durant la première phase, les répondantes se servent de stratégies passives (déni, priorisation, évitement), alors qu'elles utilisent lors des deux dernières phases, les stratégies comportementales actives, telles la recherche d'information ou de soutien social.

Des recherches ultérieures pour approfondir nos connaissances sur l'existence de ces trois phases de transition, permettraient aux intervenants et intervenantes de mieux cibler leurs interventions auprès des familles monoparentales.

\section{Supervision et gestion : essai sur le développement de modèles}

Aline A. Lévesque

École de service social, Université Laurentienne

Les stages occupent une place centrale dans la formation en service social. Dans cette formation, la supervision clinique est un travail qui demande des connaissances et habiletés particulières. Elle demande aussi une excellente compétence de communication et la capacité de développer une relation avec les divers systèmes d'un milieu de travail. Ce stage en supervision avait comme objectif l'amélioration de ces compétences et connaissances en lien avec la tâche de superviseure clinique.

Fondé sur la théorie interactionnelle de Shulman, les travaux de Munson et la théorie des systèmes, ce stage a permis à l'auteure d'évaluer la qualité des services et l'efficacité des techniques utilisées par les intervenantes dans leurs interventions auprès de la clientèle. De plus, il a permis à l'auteure de développer certains outils afin de procéder à l'évaluation de chacun des stades de leurs interventions. 


\title{
Les enjeux actuels des soins formels et informels que prodiguent les femmes
}

\author{
Michèle Mayer \\ École de service social, Université d'Ottawa
}

La présente recherche a pour but de faire le point sur les enjeux actuels des soins formels et informels que prodiguent les femmes. Pour ce faire, nous avons choisi une recherche théorique documentaire. Notre analyse de contenu des documents sélectionnés privilégie une perspective féministe pour aborder la question des soins que prodiguent les femmes.

Le contenu de notre documentation démontre que les femmes sont les principales productrices de soins et que, dans notre société, ce travail prend diverses formes durant leur vie en tant que mère, soeur, épouse, bénévole, infirmière, travailleuse sociale, etc. Malgré les exigences, la complexité et la lourdeur de ce travail, notre société tend toujours à l'occulter et les femmes s'y retrouvent trop souvent pénalisées.

Nous verrons à partir de cette recherche que les soins font l'objet de diverses définitions mais que leur conceptualisation demeure incomplète et ce, particulièrement dans le domaine des sciences sociales. Nous verrons aussi qu'en raison de leur complexité, certains auteurs ont tenté d'analyser les soins à partir d'activités bien précises qui les constituent alors que d'autres s'y sont objectés, prétendant une approche globale. Nous consacrons également la partie centrale de ce mémoire à explorer ce qui motive les femmes à soigner, car bien souvent, les conditions dans lesquelles elles le font leurs sont désavantageuses. Nous démontrerons en dernier lieu ce que l'intervention féministe peut apporter aux femmes soignantes qui vivent des difficultés. 


\section{Les personnes âgées francophones en Ontario. Un premier profil}

Jocelyne McKellar

École de service social, Université d'Ottawa

Il existe présentement dans la province de l'Ontario plus de 60000 francophones âgés de plus de 65 ans. D'après les projections démographiques, la population franco-ontarienne ne cesse de vieillir. Par contre, nous connaissons très peu les conditions de vie et de santé de cette population. Ce mémoire s'attache à brosser un portrait des conditions de vie et de santé des personnes âgées francophones en Ontario à partir de données disponibles mais fragmentées. Notre analyse vise à décrire l'environnement, le bienêtre économique et social, la santé, les habitudes de vie des personnes âgées franco-ontariennes vivant dans la communauté, en accordant une place importante aux analyses de la minorisation et aux rapports inégalitaires entre les sexes.

Pour effectuer cette étude, nous avons choisi de procéder à une analyse secondaire de données variées provenant de différentes sources, notamment les banques de données statistiques fédérales, provinciales, municipales, ainsi que les documents de presse.

Les résultats de notre analyse indiquent tout d'abord un grand besoin d'étudier les personnes âgées francophones en Ontario. Il existe d'importantes lacunes dans les études statistiques concernant cette population. Nous attribuons en partie ces lacunes au manque de visibilité des francophones âgés dans la province de l'Ontario et à leur situation minoritaire qui explique, entre autres, l'absence de connaissances à leur sujet et une vulnérabilité accrue à la pauvreté, en particulier chez les femmes.

Dans ce mémoire, nous proposons certaines pistes de réflexions sur l'intervention sociale auprès des personnes âgées francoontariennes en tenant compte de l'hétérogénéité des situations de vie qu'elles connaissent. 


\title{
La désinstitutionnalisation en santé mentale
}

\author{
Nathalie L. Ouellette \\ École de service social, Université Laurentienne
}

Depuis les trente dernières années, le système de santé mentale voit de gros changements, en particulier autour de la dépopulation des institutions psychiatriques. Les raisons varient entre le coût de ces institutions et le droit de la personne de choisir le traitement de son choix. Les personnes qui vivent avec une maladie mentale ont dû subir les conséquences d'une désinstitutionnalisation mal organisée. Ces gens ont maintenant le choix de ne pas être hospitalisés pour de longues périodes de temps; l'alternative est un manque d'accès à des services de santé mentale communautaires adéquats. Ceci engendre une certaine dépendance des expsychiatrisés(es) sur les institutions psychiatriques et ainsi un niveau très élevé de récidivisme.

Pour mieux comprendre les difficultés de la désinstitutionnalisation en santé mentale, cette recherche analyse la perspective des bénéficiaires des services psychiatriques quant à leur autonomie et leur accès à des services psychiatriques adéquats dans la communauté.

\section{Les facteurs qui amènent les femmes à faire appel à une sage femme pour leur suivi périnatal}

\author{
Sylvie Rivard \\ Ecole de service social, Université Laurentienne
}

La maternité est définie comme étant l'expérience de la grossesse et de la naissance, mais aussi de l'interprétation qui lui est donnée selon son contexte culturel et social particulier. Cette définition, tout comme l'ensemble des soins médicaux, est en évolution. 
Actuellement, la présence de sages-femmes complémente cette définition. Bien que la profession des sages-femmes existe à travers le monde depuis très longtemps, l'Ontario ne l'a intégrée légalement, à l'intérieur des soins de santé provinciaux, que depuis 1994.

L'histoire démontre comment la sage-femme possédait jadis une expertise reconnue face aux événements entourant la naissance et comment elle a perdu la reconnaissance de ses connaissances grâce à la chasse aux sorcières et au développement de la médecine - en particulier l'obstétrique. Dans la culture occidentale moderne, la médecine prend charge de définir la maternité. Cette médecine conçoit la grossesse et l'accouchement comme un état médical anormal et pathologique; d'où vient la nécessité d'intervenir pour contrôler la situation.

Le mouvement féministe et la révolution sexuelle ont initié un nouveau paradigme de la conception que les femmes ont de leur corps, et de ce fait, de la naissance. Les femmes développent une nouvelle conscience de leurs droits et de leurs responsabilités et définissent la grossesse et l'accouchement comme une étape naturelle de leur vie; une tâche que le corps maîtrise depuis des millénaires. Elles exigent alors d'exercer des choix, dont le recours à une sage-femme, pour leur suivi périnatal.

Cette étude exploratoire qualitative analyse le recours des femmes aux soins offerts par les sages-femmes pour tenter de cerner les facteurs qui les amènent à cette décision. Grâce à une analyse de contenu de douze entrevues semi-dirigées auprès de clientes des sages-femmes de la région de Sudbury, un profil de la prise de décision de ces femmes fut élaboré. Un schéma décrivant les facteurs à l'oeuvre dans la prise de décisions des répondantes ressort de cette analyse. Ceci nous permet de situer cette prise de décision dans le contexte sociologique d'un mouvement social et d'un changement de paradigme.Au centre de ce paradigme se trouvent la recherche des soins personnalisés, respectueux et humanisés, ainsi que la reconnaissance que la grossesse et l'accouchement sont des phénomènes normaux et naturels. 